\title{
Stage II Small Intestinal, Esophageal, Colorectal, Mesenteric, and Peritoneal Gastrointestinal Stromal Tumor AJCC v8
}

National Cancer Institute

\section{Source}

National Cancer Institute. Stage II Small Intestinal, Esophageal, Colorectal, Mesenteric, and Peritoneal Gastrointestinal Stromal Tumor AJCC v8. NCI Thesaurus. Code C136781.

Stage II includes: T3, NO, M0, Low Mitotic Rate. T3: Tumor measuring more than $5 \mathrm{~cm}$ but not more than $10 \mathrm{~cm}$. N0: No regional lymph node metastasis or unknown lymph node status. M0: No distant metastasis. Low Mitotic Rate: Five or fewer mitoses per 5 square millimeters, or per 50 HPF. (AJCC 8th ed.) 